\title{
The Velocity of Gravitational Waves
}

\author{
M. Novello*, V. A. De Lorenci, Luciane R. de Freitas ${ }^{\dagger}$ and O. D. Aguiar ${ }^{\ddagger}$ \\ Centro Brasileiro de Pesquisas Fúsicas, \\ Rua Dr. Xavier Sigaud, 150, Urca \\ 22290-180 - Rio de Janeiro, RJ - Brazil. \\ †Instituto de Física, \\ Universidade Federal do Rio de Janeiro \\ Ilha do Fundão - CT - Bloco A, \\ 21945 - Rio de Janeiro, RJ - Brazil. \\ $\ddagger$ Instituto Nacional de Pesquisas Espaciais, \\ Divisão de Astrofísica \\ Av. Astronautas, 1758 \\ 12227-010 - São José dos Campos, SP - Brazil.
}

\begin{abstract}
We examine the propagation of gravitational waves in new field theory of gravitation recently proposed by Novello-De Lorenci-Luciane(NDL). This examination is done on a solvable case corresponding to a spherically symmetric static configuration. We show that in NDL theory the velocity of gravitational waves is lower than light velocity. We point out some consequences of this result and suggest a possible scenario for its verification.
\end{abstract}

PACS numbers: 04.50.+h; 04.30.Nk; 04.90.+e.

* novello@lafex.cbpf.br 


\section{Introduction}

The equivalence principle (EP), which states that all kinds of matter (including massless particles such as photons) interact in the same way with the gravitational field, gave to Einstein the possibility to treat gravitational phenomena as a modification of the spacetime geometry.

Recently we have exploited some consequences of a field theoretical description of gravity with a new ingredient: we do not require that the gravitational energy satisfies the hypothesis of universality of the equivalence principle. This means that gravity does not couple to itself in the same way as all others forms of energy. The hypothesis that gravity-gravity interaction is the same as the interaction that gravity has with all other type of matter, as made in General Relativity, is not supported by direct observation, but by indirect consequences of the effect of gravitational field on matter.

The NDL theory of gravity contains many of the ingredients of GR and is indistinguishable as far as standard post-Newtonian tests and quadrupole emission are concerned (see ref. [1]). The key point to distinguish the NDL theory from GR seems to be the velocity of gravitational waves. In order to examine the behavior of such waves we will study the evolution of discontinuities of the corresponding equations of motion through a characteristic surface $\Sigma$, using the Hadamard method.

There is a general expectation concerning the possibility that we could detect gravitational waves in the next decade. Such situation is based on the great number of new experimental devices that many laboratories, throughout the world, are constructing. Many scientists are going to become involved in this enterprise. In this vein, there is a natural and direct way to test NDL theory by the analysis of the gravitational waves. We start to undertake this task in this present paper.

In the section 2 we review the NDL theory showing its formal structure. We show that the gravitational radiation propagates on a effective metric distinct from the universal metric seen by the rest of matter and the static spherically symmetric solution.

In the section 3 we make a brief review of Hadamard's method to study wave propagation.

In the section 4 we compare the velocity of electromagnetic waves in the vacuum and Gravitational waves in the NDL theory on the static spherically symmetric metric and show that in this theory the velocity of gravitational radiation is less than c [We use units of light velocity $(c=1)$ ].

\section{A Short Review of the NDL Theory of Gravity}

\subsection{General Features}

In a previous paper [1] we presented a modification of the standard Feynman-Deser approach of field theoretical derivation of Einstein's general relativity, which led to a competitive gravitational theory. The main lines of NDL approach can be summarized as follows: 
- Gravity is described by a symmetric second rank tensor $\varphi_{\mu \nu}$ that satisfies a nonlinear equation of motion;

- Matter couples to gravity in an universal way. In this interaction, the gravitational field appears only in the combination $\gamma_{\mu \nu}+\varphi_{\mu \nu}$, inducing us to define a quantity $g_{\mu \nu}=\gamma_{\mu \nu}+\varphi_{\mu \nu}$. This tensor $g_{\mu \nu}$ acts as an effective metric tensor of the spacetime as seen by matter or energy of any form except gravitational energy;

- The self interaction of the gravitational field break the universal modification of the spacetime geometry.

We define a three-index tensor $F_{\alpha \beta \mu}$, which we will call the gravitational field, in terms of the symmetric standard variable $\varphi_{\mu \nu}$ (which will be treated as the potential) to describe a spin-two field, by the expression"

$$
F_{\alpha \beta \mu}=\frac{1}{2}\left(\varphi_{\mu[\alpha ; \beta]}+F_{[\alpha} \gamma_{\beta] \mu}\right)
$$

where $F_{\alpha}$ is the trace

$$
F_{\alpha}=F_{\alpha \mu \nu} \gamma^{\mu \nu}=\varphi_{, \alpha}-\varphi_{\alpha \mu ; \nu} \gamma^{\mu \nu}
$$

From the above definition it follows that $F_{\alpha \beta \mu}$ is anti-symmetric in the first pair of indices and obeys the cyclic identity, that is

$$
\begin{gathered}
F_{\alpha \mu \nu}+F_{\mu \alpha \nu}=0 \\
F_{\alpha \mu \nu}+F_{\mu \nu \alpha}+F_{\nu \alpha \mu}=0 .
\end{gathered}
$$

In order to construct a non-linear theory for the gravitational field we make the hypothesis that gravity-gravity interaction is represented by a functional of the two invariants of the field $F_{\alpha \beta \mu}$, A and B, given by:

$$
\begin{aligned}
A & \equiv F_{\alpha \mu \nu} F^{\alpha \mu \nu}, \\
B & \equiv F_{\mu} F^{\mu} .
\end{aligned}
$$

and the Lagrangian density is a Born-Infeld type

$$
L=\frac{b^{2}}{\kappa}\left\{\sqrt{1-\frac{U}{b^{2}}}-1\right\},
$$

where $b$ is a constant and $U$ is defined by

$$
U \equiv A-B
$$

In this way, the gravitational action is expressed as:

$$
S=\int \mathrm{d}^{4} x \sqrt{-\gamma} L
$$

\footnotetext{
${ }^{1}$ We are using the anti-symmetrization symbol $[x, y] \equiv x y-y x$ and the symmetrization symbol $(x, y) \equiv x y+y x$. Note that indices are raised and lowered by the background metric $\gamma_{\mu \nu}$. The covariant derivative is denoted by a semicomma ';' and it is constructed with this metric.
} 
where $\gamma$ is the determinant of the flat spacetime metric $\gamma_{\mu \nu}$ written in an arbitrary coordinate system.

From the Hamilton principle we find the following equation of motion in the absence of material sources:

$$
\left[L_{U} F^{\lambda(\mu \nu)}\right]_{; \lambda}=0
$$

$L_{U}$ represents the derivative of the Lagrangian with respect to the invariant $U$.

As we remarked before, we follow the standard procedure [2, 3] and define an effective Riemannian metric tensor in terms of the potential $\varphi_{\alpha \beta}$, by the expression

$$
g_{\mu \nu}=\gamma_{\mu \nu}+\varphi_{\mu \nu}
$$

This relation has a deep meaning, for all forms of non-gravitational energy the net effect of the gravitational field is felt precisely as if gravity were nothing but a consequence of changing the metrical properties of the spacetime from the flatness structure to a curved one. The definition of the associated metric tensor is provided precisely by the above expression. This means that any material body (including those massless) moves along geodesics as if the metric tensor of spacetime were given by the equation (10). This is in agreement with the Equivalence Principle.

\subsection{The Static Spherically Symmetric Solution}

We set for the auxiliary metric $\left.\right|^{2}$ of the background the form

$$
\mathrm{d} s^{2}=\mathrm{d} t^{2}-\mathrm{d} r^{2}-r^{2}\left(\mathrm{~d} \theta^{2}+\sin ^{2} \theta \mathrm{d} \varphi^{2}\right) .
$$

This means that all operations of raising and lowering indices are made by this metric $\gamma_{\mu \nu}$. We remark the fact that matter[ feels a modified geometry given by Eq. (10).

A possible static spherically symmetric solution of NDL theory has only two non zero gravitational components of $\varphi^{\mu \nu}$ :

$$
\begin{aligned}
& \varphi_{00}=\varphi^{00}=\mu(r) \\
& \varphi_{11}=\varphi^{11}=-\nu(r) .
\end{aligned}
$$

In this case the gravitational field $F_{\alpha \beta \mu}$ reduces to

$$
\begin{aligned}
& F_{100}=-\frac{\nu}{r} \\
& F_{122}=\frac{F_{133}}{\sin ^{2} \theta}=\frac{1}{2} \nu r-\frac{1}{2} \mu^{\prime} r^{2}
\end{aligned}
$$

in which a prime' symbolizes the derivative with respect to the radial variable $r$. The unique component of the trace that remains is $F_{1}$,

$$
F_{1}=\mu^{\prime}-2 \frac{\nu}{r}
$$

\footnotetext{
${ }^{2}$ We note that this metric is non-observable by any form of energy.

${ }^{3}$ Massive or massless particles — photons, for instance - that is, any form of non-gravitational energy
} 
From these we can evaluate the invariant $U$ :

$$
U=\frac{\nu^{2}}{r^{2}}-\frac{2 \nu \mu^{\prime}}{r}
$$

From the equations of motion (9) we obtain

$$
\begin{gathered}
\nu=\frac{2 M}{r}\left\{1-\left(\frac{r_{c}}{r}\right)^{4}\right\}^{-\frac{1}{2}} \\
\mu=\frac{1}{2} \sqrt{b M}\left\{F(\alpha, \sqrt{2} / 2)+\mu_{0}\right\}
\end{gathered}
$$

in which the constant $r_{c}$ appearing in Eq. (19) is given by

$$
r_{c}^{2} \equiv \frac{2 M}{b}
$$

In Eq. (20) $F(\alpha, \sqrt{2} / 2)$ is the elliptic function of the first kind and the constant $\mu_{0}$ must be chosen to yield the correct asymptotic limit. The quantity $\alpha$ is given by

$$
\alpha \equiv \arcsin \left[1-\left(\frac{r_{c}}{r}\right)^{2}\right]^{\frac{1}{2}}
$$

\section{Wave Propagation}

In this section we discuss wave propagation in two steps. First we give a very intuitive example using the electromagnetic field. We make a brief review of Hadamard's method and show why in non-linear cases it is better apply this treatment. Second we use this method on the NDL theory of gravitation

\subsection{Hadamard Treatment}

Let us consider a charged particle at rest for $t \leq t_{0}$ that starts to be accelerated in $t=t_{0}$. The electromagnetic field produced by this particle was initially independent of time and after that it becomes a variable field. Hence, there are two regions of space-time one in which the field is independent of the time and one in which the field varies. These regions are separated by a hypersurface $\Sigma$. As the accelerated particle emits radiation, the hypersurface $\Sigma$ is the corresponding wavefront.

Mathematically such a hypersurface $\Sigma$ is characterized by the fact that some derivatives of the electromagnetic potential $A_{\mu}$ are discontinuous across $\Sigma$. This effect comes from the fact that all derivatives of the potential $A_{\mu}$ are zero in the region $\Sigma^{-}$but not in $\Sigma^{+}$. These disturbances are propagated by the equation of motion.

Although the situation described above is a good image for any field disturbance, the equation of motion of the gravitational field is non-linear and this fact causes a great difference between the analysis of the propagation of a gravitational wave and of an electromagnetic wave. Due to this, the better and simpler method to study the propagation of the gravitational wave is Hadamard's method, which we explain briefly in the following. 
Let us define a hypersurface of discontinuity $\Sigma$ by the equation:

$$
\Sigma: \varphi\left(x^{\alpha}\right)=0 .
$$

The space-time is divided in two regions: $\Sigma^{+}$(where $\varphi>0$ ) and $\Sigma^{-}$(where $\varphi<0$ ). The discontinuity of any function $f\left(x^{\alpha}\right)$ across $\Sigma$ is defined by:

$$
\left[f\left(x^{\alpha}\right)\right]=\lim _{p^{ \pm} \rightarrow p}\left(f\left(P^{+}\right)-f\left(P^{-}\right)\right)
$$

where $P^{+}$is in $\Sigma^{+}$and $P^{-}$is in $\Sigma^{-}$. To introduce the algebraic formalism proposed by Hadamard we use the definition of the total differential of a function $f$, to state that

$$
[d f]=0
$$

since

$$
[d f]=\lim _{p^{ \pm} \rightarrow p}\left(d f\left(P^{+}\right)-d f\left(P^{-}\right)\right)
$$

It follows

$$
[d f]=\left[\frac{\partial f}{\partial x^{\alpha}} d x^{\alpha}\right]=0
$$

but $x^{\alpha}$ is a coordinate and since the coordinate system is continuous, $d x^{\alpha}$ is also, hence,

$$
\left[\frac{\partial f}{\partial x^{\alpha}}\right] d x^{\alpha}=0
$$

this differential is calculated on the surface therefore $d x^{\alpha}$ is parallel to it

$$
\left[\frac{\partial f}{\partial x^{\alpha}}\right] \propto \varphi_{, \alpha} \equiv K_{\alpha}
$$

where $\varphi_{, \alpha}$ is normal to hypersurface $\Sigma$. This result is valid for tensorial functions of any rank. We can verify this using an suitable transformation of coordinates for a particular case of a vector and it is straightforward extended to higher rank tensor.

\subsection{Wave Propagation in NDL Theory}

Let us review briefly the result set up in [1] for the velocity of the gravitational wave in NDL theory. We represent by the symbol $[J]_{\Sigma}$ the discontinuity of the function $J$ across the surface $\Sigma$. Following Hadamard we impose the discontinuity conditions for the field:

$$
\left[F_{\mu \nu \alpha}\right]_{\Sigma}=0
$$

and

$$
\left[F_{\mu \nu \alpha ; \lambda}\right]_{\Sigma}=f_{\mu \nu \alpha} k_{\lambda},
$$

where $k_{\alpha}$ represents the normal vector to the surface of discontinuity $\Sigma$. $f_{\alpha \beta \gamma}$ has the same symmetries as the field $F_{\alpha \beta \gamma}$. Taking the discontinuity of the equation of motion (9) we obtain:

$$
f^{\mu}{ }_{(\alpha \beta)} k_{\mu}+2 \frac{L_{U U}}{L_{U}}(\eta-\zeta) F^{\mu}{ }_{(\alpha \beta)} k_{\mu}=0
$$


in which we defined $\eta \equiv F_{\alpha \beta \mu} f^{\alpha \beta \mu}$ and $\zeta \equiv F_{\mu} f^{\mu}$. In the same way, taking the discontinuity of the indentity

$$
F_{\alpha \beta_{; \gamma}{ }^{\lambda}+F_{\beta \gamma}{ }^{\lambda} ; \alpha}+F_{\gamma \alpha}{ }^{\lambda} ; \beta=\frac{1}{2} \delta_{[\alpha}^{\lambda} F_{\gamma] ; \beta}+\frac{1}{2} \delta_{[\gamma}^{\lambda} F_{\beta] ; \alpha}+\frac{1}{2} \delta_{[\beta}^{\lambda} F_{\alpha] ; \gamma}
$$

contracted with $F^{\alpha \beta \lambda} k^{\gamma}$, we obtain:

$$
(\eta-\zeta) k^{2}+2 F^{\alpha \beta \lambda} f_{\beta \gamma \lambda} k_{\alpha} k^{\gamma}+F^{\alpha \beta \lambda} f_{\alpha} k_{\beta} k^{\lambda}+F^{\beta} f^{\gamma} k_{\beta} k^{\gamma}=0 .
$$

Working with equations (31) and (33), we arise at the following expression for the propagation:

$$
k_{\mu} k_{\nu}\left[\gamma^{\mu \nu}+\Lambda^{\mu \nu}\right]=0
$$

in which the quantity $\Lambda^{\mu \nu}$ is written in terms of the gravitational field:

$$
\Lambda^{\mu \nu} \equiv 2 \frac{L_{U U}}{L_{U}}\left[F^{\mu \alpha \beta} F_{(\alpha \beta)}^{\nu}-F^{\mu} F^{\nu}\right]
$$

Note that the discontinuities of the gravitational fields propagate in a modified geometry, changing the background geometry $\gamma^{\mu \nu}$ into an effective one $g_{\text {eff }}^{\mu \nu}$,

$$
g_{\text {eff }}^{\mu \nu} \equiv \gamma^{\mu \nu}+\Lambda^{\mu \nu}
$$

which depends on the field $F_{\alpha \beta \mu}$ and also on the dynamics. This fact shows that such a property stems from the structural form of the Lagrangian. Thus, in the NDL theory the characteristic surfaces of the gravitational waves propagate on the null cone of an effective geometry. We remark that this geometry is distinct from that observed by all other forms of energy and matter, which differs from the general relativity predictions. This result gives a possibility to choose between these two theories just by observations of the gravitational waves. In the next section we will present a summary of the main properties of the solutions of the gravitational field in NDL theory. Then we will evaluate the velocity of gravitational wave in such background and compare with the GR result.

\section{Electromagnetic and Gravitational Waves in the Spherically Symmetric Solution}

From what we have seen, in the NDL theory all kinds of matter and non-gravitational energy couple to gravity differently from gravity to gravity interaction. In order to compare the propagation of electromagnetic and gravitational waves we will proceed as follows.

The metric that defines the structure of the spacetime in which the discontinuities of the electromagnetic field propagate, is provided by Eq. (10). We define a 4 -vector $l_{\mu}$ such that

$$
l_{\mu} l_{\nu} g^{\mu \nu}=0
$$

where $g^{\mu \nu}$ is the inverse of the metric $g_{\mu \nu}$, defined by

$$
g^{\mu \rho} g_{\mu \sigma}=\delta_{\sigma}^{\rho} .
$$


Analogous to the section (3), this 4-vector is a gradient of the hypersurface of discontinuities. The background gravitational field we are considering here, corresponds to a spherically symmetric and static configuration. Thus, the relation (37) reduces in this case to

$$
\frac{\left(l_{0}\right)^{2}}{1+\mu}-\frac{\left(l_{1}\right)^{2}}{1+\nu}-\frac{\left(l_{2}\right)^{2}}{r^{2}}-\frac{\left(l_{3}\right)^{2}}{r^{2} \sin ^{2} \theta}=0
$$

Gravitational waves propagate as null geodesic in an effective geometry $g_{\text {eff }}^{\mu \nu}$ given by Eq. (36). The quantity $\Lambda^{\mu \nu}$ is defined by Eq. (35) and have only two non null components:

$$
\begin{aligned}
& \Lambda^{00}=-\frac{4 M^{2}}{b^{2} r^{4}} \\
& \Lambda^{11}=-\Lambda^{00} .
\end{aligned}
$$

Correspondingly, the effective metric is provided by

$$
\begin{aligned}
g_{\mathrm{eff}}^{00} & =1-\frac{4 M^{2}}{b^{2} r^{4}} \\
g_{\mathrm{eff}}^{11} & =-1+\frac{4 M^{2}}{b^{2} r^{4}} \\
g_{\mathrm{eff}}^{22} & =-\frac{1}{r^{2}} \\
g_{\mathrm{eff}}^{33} & =-\frac{1}{r^{2} \sin ^{2} \theta}
\end{aligned}
$$

Inserting these results in Eq. (34) the equation of propagation of gravitational waves became:

$$
\left(1-\frac{4 M^{2}}{b^{2} r^{4}}\right)\left(k_{0}\right)^{2}-\left(1-\frac{4 M^{2}}{b^{2} r^{4}}\right)\left(k_{1}\right)^{2}-\frac{\left(k_{2}\right)^{2}}{r^{2}}-\frac{\left(k_{3}\right)^{2}}{r^{2} \sin ^{2} \theta}=0 .
$$

We can summarize this situation as:

- Electromagnetic waves propagate in null cone of the geometry $g_{\mu \nu}$;

- Gravitational waves propagate in null cone of the geometry $g_{\mu \nu}^{\text {eff }}$.

A question then arises: Which wave propagates faster? In order to investigate this problem we can run by two equivalent ways: one can either evaluate the norm of the vector $l_{\mu}$ in the geometry $g_{\mu \nu}^{\text {eff }}$ :

$$
l_{\mu} l_{\nu} g_{\text {eff }}^{\mu \nu}
$$

or else evaluate the norm of the vector $k_{\mu}$ in the geometry $g_{\mu \nu}$ :

$$
k_{\mu} k_{\nu} g^{\mu \nu}
$$

In this vein we evaluate the quantity (47) and investigate the character of $l_{\mu}$ in the geometry $g_{\text {eff }}^{\mu \nu}$. For the solution we are considering here, results:

$$
\|l\|_{g_{\mathrm{eff}}}=\left(1-\frac{4 M^{2}}{b^{2} r^{4}}\right)\left(l_{0}\right)^{2}-\left(1-\frac{4 M^{2}}{b^{2} r^{4}}\right)\left(l_{1}\right)^{2}-\frac{\left(l_{2}\right)^{2}}{r^{2}}-\frac{\left(l_{3}\right)^{2}}{r^{2} \sin ^{2} \theta}
$$


Substituting expression (39) in the last two terms in the right hand side of the above equation, we obtain

$$
\|l\|_{g_{\mathrm{eff}}}=\left(1-\frac{4 M^{2}}{b^{2} r^{4}}-\frac{1}{1+\mu}\right)\left(l_{0}\right)^{2}-\left(1-\frac{4 M^{2}}{b^{2} r^{4}}-\frac{1}{1+\nu}\right)\left(l_{1}\right)^{2} .
$$

The solutions of the functions $\mu$ and $\nu$ are given by (20) and (19). Since we are interested here just in the sign of the norm of the 4 -vector $l_{\mu}$ in the geometry determined by $g_{\mu \nu}^{\text {eff }}$, it is enough to consider only the main terms. Thus, expanding the field solutions as:

$$
\begin{aligned}
& \frac{1}{1+\mu} \approx 1+\frac{2 M}{r}+O\left(r^{-2}\right) \\
& \frac{1}{1+\nu} \approx 1-\frac{2 M}{r}+O\left(r^{-2}\right),
\end{aligned}
$$

and substituting in Eq. (50) result in

$$
\|l\|_{g_{\mathrm{eff}}}=-\frac{2 M}{r}\left(l_{0}\right)^{2}-\frac{2 M}{r}\left(l_{1}\right)^{2} .
$$

Hence, $\|l\|_{g_{\text {eff }}}<0$. We thus conclude that $l_{\mu}$ is a space-like vector in the geometry $g_{\mu \nu}^{\text {eff }}$, i.e., in the NDL theory gravitational waves travel with velocity lower than light.

\section{Conclusion}

The NDL theory prediction that gravitational waves travel slower than light has some interesting consequences. The first one is the possibility to exist gravitational Chêrenkov Radiation, that is the emission of gravitational radiation when a massive particle exceeds locally the "graviton" speed [5]. Evidently, this phenomenon will put limits on how far from their sources cosmic rays can be found with ultra high energies, such as the ones searched by the AUGER Project $\left(\mathrm{E} \geq 10^{20} \mathrm{eV}\right)$ [6]. How stringent these limits are will be addressed in future work.

One question naturally stands up: can we obtain observational evidence that gravitational waves travel at speeds below the light velocity in the presence of gravitational potentials? Very high gravitational potentials can be found in the vicinities of neutron stars, black holes and supernova cores. However, unless we are talking about very massive black holes, all these gravitational sources are not sufficiently extended in size to allow the integration of a large enough difference in the arriving times at Earth for photons and gravitons supposely generated at the same place and instant. On the other hand, black holes of $10^{6}$ to $10^{9}$ solar masses, as it is believed exist at the center of galaxies such as M87, M51 and others [7], present much better conditions to delay the gravitational waves relatively to the electromagnetic waves. After all, the Schwarzschild radius of a $10^{9}$ solar mass black hole has the size of two Astronomical Units, a distance that light takes about 1000 seconds to cross if traveling outside the horizon. This may give us enough time for the potential to act on the gravitational waves, slowing them down for sufficient time, in order to accumulate a time delay possible to be measured with the technology of the next generations of gravitational wave observatories and electromagnetic telescopes in the few decades to come. 


\section{Acknowledgements}

We would like to thank the participants of the "Pequeno Seminário" of Lafex/CBPF. This work was supported by "Conselho Nacional de Desenvolvimento Científico e Tecnológico" (CNPq) and "Fundação de Amparo a Pesquisa no Estado do Rio de Janeiro" (FAPERJ) of Brazil.

\section{References}

[1] M. Novello, V.A. De Lorenci and L.R. de Freitas, Annals of Phys., 254, $n^{o}$ 1, 83 (1997); M. Novello and V. A. De Lorenci, report no. gr-qc/9704025.

[2] R.P.Feynman, in Feynman Lectures On Gravitation, (Addison-Wesley Pub. Company, Massachusetts, 1995).

[3] S.Deser, J. Gen. Rel. Grav., 1, 9, (1970).

[4] J. Hadamard, in Lectures on Cauchy's Problem (Yale University Press 1923); Dover reprint (1952).

[5] C.M.Caves, Annals of Phys., 125, 35 (1980).

[6] C.O.Escobar, in Proceedings of the 7th International Symposium on Radiation Physics, Jaipur, India, Feb/97. Ed. D. Bradley.

[7] V.L.Ginzburg and L.M.Ozernoy, Astrophysics and Space Science, 50, 23 (1977). 